\title{
Polish trials influencing 2017 European Society of Cardiology guidelines on acute myocardial infarction in patients presenting with ST-segment elevation
}

\author{
Małgorzata Ostrowska, Piotr Adamski \\ Department of Principles of Clinical Medicine, Collegium Medicum in Bydgoszcz, Nicolaus Copernicus University in Torun, Poland
}

Adv Interv Cardiol 2017; 13, 3 (49): 189-190 DOI: https://doi.org/10.5114/aic.2017.70185

During the latest annual European Society of Cardiology Congress in Barcelona, new guidelines for the management of acute myocardial infarction in patients presenting with ST-segment elevation (STEMI) were presented [1]. It is noteworthy that, apart from international, multicenter studies involving sites from Poland, the results of three Polish trials had a significant impact on the shape of the current recommendations on the management of STEMI patients [2-4].

Alleviation of chest pain is one of the main therapeutic targets in patients presenting with STEMI, and titrated intravenous morphine is a routinely administered analgesic in this setting. However, a recent paper by Kubica et al. revealed that morphine delays and attenuates ticagrelor exposure and action in patients with myocardial infarction [2]. The randomized, double-blind, placebo-controlled IMPRESSION trial, conducted in the Department of Cardiology and Internal Medicine, Collegium Medicum, Nicolaus Copernicus University in Bydgoszcz, aimed to evaluate the influence of infused morphine on pharmacokinetics and pharmacodynamics of ticagrelor and its active metabolite in patients with acute myocardial infarction. Seventy patients were assigned in a $1: 1$ ratio to receive either morphine $(5 \mathrm{mg}$ ) or placebo intravenously followed by a $180 \mathrm{mg}$ loading dose of ticagrelor. Morphine lowered the total exposure to ticagrelor and its active metabolite by $36 \%$ (AUC $_{(0-12)}: 6307$ vs. $9791 \mathrm{ng}$ $\left.{ }^{*} \mathrm{~h} / \mathrm{ml} ; p=0.003\right)$ and $37 \%\left(\mathrm{AUC}_{(0-12)}\right): 1503$ vs. $2388 \mathrm{ng}$ * $\mathrm{h} / \mathrm{ml} ; p=0.008)$, respectively. Moreover, a delay in maximal plasma concentration of ticagrelor (4 vs. 2 h; $p=0.004$ ) was observed in patients receiving morphine. Multiple regression analysis showed that lower $\mathrm{AUC}_{(0-12)}$ values for ticagrelor were independently associated with the administration of morphine $(p=0.004)$ and the presence of STEMI $(p=0.014)$. In pharmacodynamic assess- ment up to three platelet reactivity tests were used - the vasodilator-stimulated phosphoprotein phosphorylation assay, multiple electrode aggregometry and VerifyNow and all of them revealed a stronger antiplatelet effect in the placebo group and a greater prevalence of high platelet reactivity in the morphine arm. Despite the results of the IMPRESSION study clearly showing a negative impact of morphine on bioavailability and antiplatelet action of ticagrelor, due to lack of effective alternative, titrated intravenous opioids should be considered to relieve pain in STEMI patients. Nevertheless, the class of recommendation for use of morphine in STEMI has been lowered from I to lla with a level of evidence $C[1,5]$. In everyday clinical practice morphine should not be routinely used in all STEMI patients together with antiplatelet agents, but this decision should be made individually after thorough evaluation to ensure that morphine is restricted to patients who actually need it $[1,2,6]$.

It is a clear recommendation that reperfusion therapy is indicated in all STEMI patients with symptoms of ischemia lasting less than 12 h; however, it is still a class I recommendation to perform primary percutaneous coronary intervention $(\mathrm{PCl})$ also in those with symptom onset $>12 \mathrm{~h}$, but $<24 \mathrm{~h}$ and presence of ongoing symptoms suggestive of ischemia, hemodynamic instability, or life-threatening arrhythmias [1]. Evidence for such an approach comes from a prospective national observational study (PL-ACS) published by Gierlotka et al. from the Silesian Center of Heart Diseases in Zabrze [3]. The aim of this study was to evaluate whether primary $\mathrm{PCl}$ improves 12-month survival in those presenting with STEMI between 12 to $24 \mathrm{~h}$ from the onset of symptoms. The analyzed data concerned 2036 patients included in the Polish Registry of Coronary Syndromes. Patients with pulmonary edema, cardiogenic shock or initially treated

\section{Corresponding author:}

Małgorzata Ostrowska MD, PhD, Department of Principles of Clinical Medicine, Collegium Medicum, Nicolaus Copernicus University, 9 Skłodowskiej-Curie St, 85094 Bydgoszcz, Poland, phone: +48 5258540 23, fax: +48 5258540 24, e-mail: ostrowska.go@gmail.com Received: 12.09.2017, accepted: 17.09.2017. 
with thrombolysis were excluded from the investigation. Coronary angiography was performed within 12 to $24 \mathrm{~h}$ from symptoms onset in 910 cases (44.7\%), and $92.0 \%$ of them underwent primary $\mathrm{PCI}$. Patients qualified for an invasive strategy had a lower mortality rate after 12 months than those treated with a conservative strategy $(9.3 \%$ vs. $17.9 \% ; p<0.0001)$. Multivariate adjustment confirmed the benefit of an invasive strategy with a relative risk of 0.73 for 12 -month mortality (95\% confidence interval: $0.56-0.96)$. In conclusion, up to $10 \%$ of patients with STEMI present 12 to $24 \mathrm{~h}$ from the symptom onset and they should be considered for reperfusion by primary $\mathrm{PCl}$, because an invasive strategy reduces the 12-month mortality rate as compared to a conservative strategy in this subpopulation of latecomers. The class of recommendation for such management has changed from $\mathrm{llb}$ to lla with the level of evidence $C[1,5]$.

Multivessel coronary artery disease is frequently found in coronary angiography in STEMI patients [1]. The aim of the study by Dziewierz et al., from the $2^{\text {nd }}$ Department of Cardiology, Collegium Medicum, Jagiellonian University in Krakow, was to evaluate the influence of multivessel coronary artery disease and noninfarct-related artery revascularization during the index $\mathrm{PCl}$ on outcomes of STEMI patients [4]. The authors analyzed data of 1598 patients with multivessel $(\geq 1)$ coronary artery disease enrolled in the EUROTRANSFER Registry database. Out of identified patients, $51.5 \%$ of STEMI patients had a multivessel disease $-32 \%$ had 2-vessel disease and $19.5 \%$ had 3-vessel disease. Final Thrombolysis In Myocardial Infarction grade 3 flow was found in $93.6 \%$ of patients with 1-vessel disease, $89.3 \%$ in 2-vessel disease and $87.9 \%$ in 3 -vessel disease $(p=0.003)$. ST-segment resolution $>50 \%$ within 60 min after $\mathrm{PCl}$ occurred in $80.9 \%$ of patients with 1 -vessel disease, $77.5 \%$ in 2-vessel disease and $69.3 \%$ in 3 -vessel disease $(p<0.001)$. Risk of 1 -year death was the lowest in 1 -vessel disease (4.9\%), moderate in 2-vessel disease (7.4\%) and the highest in 3-vessel disease $(13.5 \%)$ ( $p<0.001)$. In multivariate regression analysis 1-year mortality predictors besides multivessel disease were Killip class IV on admission and left anterior descending coronary artery as infarct-related vessel. Only $9 \%$ of patients had noninfarct-related artery revascularization during the index $\mathrm{PCl}$, and they were at higher risk of both 30-day and 1-year mortality as compared with multivessel disease patients without noninfarct-related artery $\mathrm{PCl}$. To conclude, patients with STEMI commonly present with multivessel disease (> 50\%) and in the study by Dziewierz et al. they had a worse prognosis as compared with 1-vessel disease, which was even poorer when the noninfarct-related artery revascularization during index $\mathrm{PCl}$ was performed. Guidelines recommend noninfarct-related artery revascularization in patients with STEMI and multivessel disease before the hospital discharge (class of recommendation Ila, level of evidence A), while noninfarct-related artery revascularization during the index $\mathrm{PCl}$ should be reserved for those with cardiogenic shock (class of recommendation Ila, level of evidence $C)$ [1].

In the recently published 2017 guidelines for the management of STEMI patients there are only three citations originating from Poland [1-4]. The impact of these studies was significant enough to influence the change in class of recommendation in the described clinical situations.

\section{Conflict of interest}

The authors declare no conflict of interest.

\section{References}

1. The Task Force for the management of acute myocardial infarction in patients presenting with ST-segment elevation of the European Society of Cardiology (ESC). Task Force Members: Ibanez B, James S, Agewall S, et al. 2017 ESC Guidelines for the management of acute myocardial infarction in patients presenting with ST-segment elevation. Eur Heart J 2017 [Epub ahead of print]; doi:10.1093/eurheartj/ehx393.

2. Kubica J, Adamski P, Ostrowska M, et al. Morphine delays and attenuates ticagrelor exposure and action in patients with myocardial infarction: the randomized, double-blind, placebo-controlled IMPRESSION trial. Eur Heart J 2016; 37: 245-52.

3. Gierlotka M, Gasior M, Wilczek K, et al. Reperfusion by primary percutaneous coronary intervention in patients with ST-segment elevation myocardial infarction within 12 to 24 hours of the onset of symptoms (from a prospective national observational study [PL-ACS]). Am J Cardiol 2011; 107: 501-8.

4. Dziewierz A, Siudak Z, Rakowski T, et al. Impact of multivessel coronary artery disease and noninfarct-related artery revascularization on outcome of patients with ST-elevation myocardial infarction transferred for primary percutaneous coronary intervention (from the EUROTRANSFER Registry). Am J Cardiol 2010; 106: 342-7.

5. Task Force on the management of ST-segment elevation acute myocardial infarction of the European Society of Cardiology (ESC). Steg PG, James SK, Atar D, et al. ESC Guidelines for the management of acute myocardial infarction in patients presenting with ST-segment elevation. Eur Heart J 2012; 33: 2569-619.

6. Adamski P, Adamska U, Ostrowska M, et al. New directions for pharmacotherapy in the treatment of acute coronary syndrome. Expert Opin Pharmacother 2016; 17: 2291-306. 